Check for updates

Cite this: RSC Adv., 2018, 8, 24470

\title{
Apigenin ameliorates vascular injury in rats with high fructose-induced metabolic disturbance by inhibiting PI3K/AKT/GLUT1
}

\author{
Xiaofang Chen, $\uparrow$ Jianyang Tan, $\uparrow$ Lu Zhang, Yonggang Liu, Yahong Cheng, \\ Qianying Zhang and Hong Ding (D) *
}

\begin{abstract}
The abuse of fructose in daily diet may cause cardiovascular diseases that seriously threaten human health, and both safe and efficient solutions need to be developed. We investigated whether apigenin can prevent the harmful impact of excessive fructose on cardiovascular events. Based on the reduction of percentage of body fat and systolic pressure as well as the improvements in insulin resistance, lipid metabolism, and pathological injury to the thoracic aorta, we suggested that high levels of fructose cause vascular injury and metabolic disorders, which can be improved to some extent by using apigenin. Fundamentally, apigenin down-regulates levels of phosphoinositide 3-kinase (PI3K), protein kinase B (AKT), and glucose transporter 1 (GLUT1), which increase with high concentrations of fructose. Moreover, the inflammation and asymmetric dimethylarginine (ADMA) levels increased in fructose group, but they decreased when the rats were fed with apigenin. The results suggest that PI3K/AKT/GLUT1 may have potential for alleviating cardiovascular injury, and apigenin can be an excellent candidate for supplements to

ameliorate cardiovascular diseases related to high fructose consumption.
\end{abstract}

Received 25th May 2018

Accepted 21st June 2018

DOI: $10.1039 / \mathrm{c} 8 \mathrm{ra0} 4459 \mathrm{~g}$

rsc.li/rsc-advances

\section{Introduction}

With the rapid development in agricultural biotechnologies and the extensive enrichment of food supply, excessive absorption of fructose in our daily life has been an increasingly inevitable concern for the modern society. In previous research, fructose was reported to trigger a series of diseases, e.g., non-alcoholic fatty liver, pulmonary arterial hypertension, metabolic syndrome, and cardiovascular disorders; ${ }^{1-3}$ these diseases negatively affect the quality of life and human health, which is becoming an urgent problem for the medical community. However, it is universally accepted that compared to other kinds of carbohydrates, fructose is less harmful to human health; thus, fructose has been extensively used in the food industry in the form of crystalline fructose or high fructose syrup to elevate the sweetness of the food, which causes great negative impacts on human health..$^{4,5}$ Therefore, when humans are exposed to excessive fructose through daily diet, both safe and effective solutions need to be explored extensively to prevent cardiovascular diseases.

When the body receives excessive carbohydrates, different glucose transporter (GLUT) subtypes play important roles in the

Key Laboratory of Combinatorial Biosynthesis and Drug Discovery, Ministry of Education, Wuhan University School of Pharmaceutical Sciences, Wuhan University, Wuhan 430071, Hubei, P. R. China. E-mail: dinghong1106@whu.edu.cn; Fax: $+862768759963$

$\dagger$ Xiao fang Chen and Jian yang Tan contributed equally to this work. regulation of the transport of glucose or fructose to various tissues, which affects the development of health and causes various diseases. ${ }^{6-8}$ For instance, glucose transporter-1 (GLUT1), which exists mainly in erythrocytes, is a valuable marker that can be used to identify malignant tumors in patients. ${ }^{9}$ Moreover, phosphatidylinositol 3-kinase (PI3K) and protein kinase B (AKT) can affect the expression and location of downstream proteins to participate in the process of metabolism..$^{10,11}$ In addition, asymmetric dimethyl arginine (ADMA) may further increase the severity of endothelial dysfunction in the body. ${ }^{12}$ However, superfluous intake of fructose may lead to metabolic disorders and other complications in mice. ${ }^{13,14}$

Flavonoids extracted from plants and vegetables have potential positive effects on the prevention of metabolic diseases such as cancer, cardiovascular diseases and diabetes. ${ }^{15-17}$ Apigenin (5,7,4-trihydroxyflavone) is a natural flavone, and it is found extensively in some condiments and many vegetables such as celery, onions, oranges, and peppers. ${ }^{18,19}$ The chemical structure of apigenin is shown in Fig. 1A. ${ }^{20}$ Apigenin may provide protection against the dysfunction of vascular endothelium, but very few studies have been carried out on the injury of blood vessels induced by high concentrations of fructose. ${ }^{21,22}$

We designed this study to evaluate whether apigenin can relieve the negative impacts of vascular injury caused by superfluous fructose intake in Sprague Dawley (SD) rats. In addition, our research also explored whether the beneficial effect of apigenin in a high fructose environment was associated 


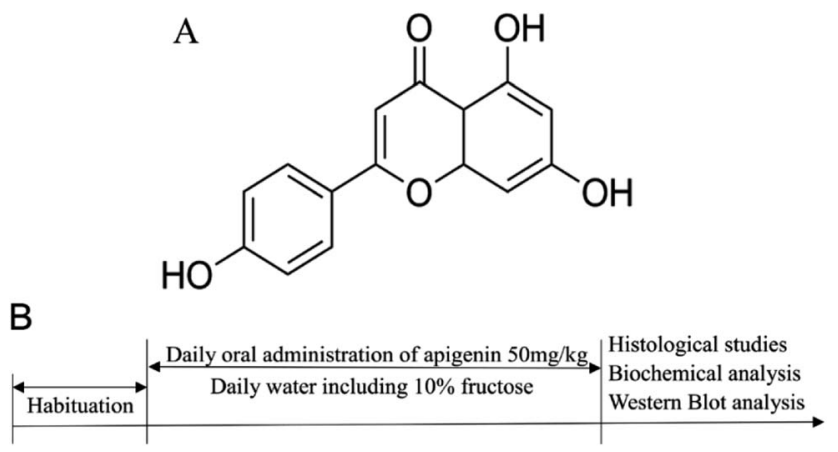

Fig. 1 (A) Chemical structure of apigenin. (B) Scheme depicting the experimental design for the time course of fructose and apigenin administration and the timelines for the histological studies, biochemical analysis and western blot analysis.

with the inhibition of PI3K/AKT/GLUT1 signalling pathway and ADMA level. Overall, the research provided insights into the potential of an ideal supplement to ameliorate cardiovascular diseases caused by high fructose in our daily diet; we focused on the possible mechanisms to provide new targets for avoiding the damage caused by excessive consumption of fructose.

\section{Materials and methods}

\section{Chemicals and materials}

D-Fructose (purity 99\%) was procured from Aladdin Chemistry Co. (Shanghai, China) and apigenin (purity 98\%) was obtained from Adamas Reagent Co., Ltd (Shanghai, China). Triglyceride (TG), total cholesterol (TC) and high-density lipoprotein cholesterol (HDL-C) kits were purchased from Jiancheng Bioengineering Institute (Nanjing, China). Glucose assay kit was obtained from Rongsheng Biological Pharmaceutical Co. (Shanghai, China). Elisa kits made by Wuhan ColorfulGene Biological Technology Co. (Wuhan, China) were used. AntiPI3K, anti-AKT and anti-GLUT1 antibodies were obtained from Proteintech Group (Chicago, USA). Goat anti-mouse IgG and goat anti-rabbit IgG conjugated to horseradish peroxide were the products of Boster (Wuhan, China).

\section{Animals and treatments}

In the experiment, male Sprague Dawley (SD) rats were purchased from Wuhan University Laboratory Animal Center; at the beginning of the experiment, their body weights ranged from $180 \mathrm{~g}$ to $220 \mathrm{~g}$ ( 7 to 8 weeks old). The rats were kept in a drafty room, where the relative humidity was maintained between $60 \%$ and $80 \%$ with a $12 \mathrm{~h}$ light/dark cycle at $25 \pm 2{ }^{\circ} \mathrm{C}$.

After acclimating for 5 days with free access to normal forage and drinking water, SD rats were randomly divided into three groups $(N=8)$ : normal control group, $10 \%$ fructose model group, and $10 \%$ fructose + apigenin $\left(50 \mathrm{mg} \mathrm{kg}^{-1}\right)$ group. ${ }^{23-25}$ The concentration of apigenin $\left(50 \mathrm{mg} \mathrm{kg}^{-1}\right)$ was determined using a preliminary unpublished study in our laboratory. The metabolic disorders were induced in all groups except the normal control group by oral administration of $10 \%$ fructose in the drinking water for twelve weeks consistently. Apigenin (50 mg $\mathrm{kg}^{-1}$ ) was administered to the apigenin group by gavage once a day regularly for twelve weeks. Rats of all the groups were fed normal feedstuff every day. All the experiments were performed in accordance with the procedure shown in Fig. 1B.

All animal procedures were performed following the National Institute of Health Guide for the Care and Use of Laboratory Animals. Also, all animal experiments were performed in consonance with the guidelines on ethical use and care by "The Institutional Animal Care and Use Committee (IACUC), Wuhan University Center for Animal Experiment", Wuhan, China.

\section{Percentage of body fat and blood pressure}

The body weight of rats was recorded once a week throughout the experiment. When the rats were sacrificed, white adipose tissues around the kidney and testis were collected to weigh for analysis of the percentage of body fat. The systolic blood pressure (SBP) was measured when rats were in quiescent condition by the tail-cuff plethysmography blood pressure analysis system (Taimeng Scientific and Technologic Co., Ltd, Chengdu, China). The final result was calculated from the mean of five continuous measurements.

\section{Measurement of insulin resistance}

The levels of insulin and glucose in serum were determined by the ELISA kits when the rats were in a fasting condition for 8 hours. Homeostasis model assessment of insulin resistance (HOMA-IR) and Quantitative Insulin Sensitivity Check Index (QUICK) were used to evaluate the resistance and sensitivity for insulin. ${ }^{26}$ Final concentrations of blood glucose were multiplied by the concentration of insulin and then divided by 22.5 to get HOMA-IR for insulin resistance. ${ }^{27}$

\section{Measurement of serum lipid level}

The blood samples $(N=8)$ were centrifuged at $10000 \mathrm{~g}$ for 8 minutes at $4{ }^{\circ} \mathrm{C}$ as soon as possible and then, we collected the supernatant carefully. The serum was divided into several aliquots for different indexes in experiments carried out at different times. The levels of triglyceride (TG), total cholesterol (TC) and high-density lipoprotein cholesterol (HDL-C) were measured as the indexes of dyslipidemia with reagent kits according to the directions. ${ }^{28}$

\section{Histopathological analyses of thoracic aorta}

After the anatomical experiment, the middle part of the thoracic aorta was fixed in $4 \%$ paraformaldehyde for more than 48 hours. Then, these samples were routinely embedded in paraffin and sliced into serial sections. The sections were stained with hematoxylin-eosin (HE) and Elastica van Gieson (EVG) in accordance with general routine. EVG staining presented the change of elastic fibers of the thoracic aorta. We used the Image pro plus software to measure the thickness of the thoracic aorta. HE staining was used to analyse the histology and morphology of thoracic aorta under a microscope (Olympus). 


\section{Western blot analysis}

During experiments, thoracic aorta samples were homogenized with pre-cooling lysis buffer including $0.1 \mathrm{mM}$ phenyl-methane sulfonyl fluoride (PMSF) on ice. Aliquots of $60 \mu \mathrm{g}$ proteins were prepared for $10 \%$ sodium dodecyl sulfate (SDS) polyacrylamide gel and then, they were transferred onto $0.45 \mu \mathrm{m}$ polyvinylidene fluoride (PVDF) membranes. The membranes were covered completely by $5 \%$ bull serum albumin (BSA) for thirty minutes at $37^{\circ} \mathrm{C}$. After that, the membranes were fully soaked in primary antibodies at $4{ }^{\circ} \mathrm{C}$ overnight including PI3K (dilution $1: 500$ ), AKT (dilution $1: 100$ ), GLUT1 and $\beta$-actin (dilution $1: 500$ ) antibodies. After three washes with Tris-buffered saline Tween20 (TBST) for five minutes each time, the membranes were incubated with horseradish peroxidase-conjugated secondary antibodies (dilution $1: 5000$ ) for 1 hour at room temperature. The blots were washed three times again and then, the binding reaction signal was revealed by a chemiluminescence system to compare the relative expressions.

\section{Immunofluorescence staining}

Immunofluorescence staining was performed after the frozen experiment of thoracic aorta. Bull serum albumin (5\%) blocked the nonspecific binding sites. Further incubation was implemented with rabbit polyclonal anti-GLUT1 antibody (dilution $1: 300$ ) at $4{ }^{\circ} \mathrm{C}$ overnight. After three washings with PBS for five minutes each time, the tissues were incubated with FITCconjugated goat anti-rabbit IgG (dilution $1: 1000$ ) for 1 hour at room temperature. After washing three times again, the tissues were stained at room temperature for 10 minutes with 4',6-diamidino-2-phenylindole (DAPI). Then, the slides were evaluated with a microscope equipped with a charge-coupled device camera system (OLYMPUS, Japan).

\section{ADMA level determined by ELISA}

The level of asymmetrical dimethylarginine (ADMA) in the serum was measured by an ELISA kit in accordance with the instructions. ADMA was measured as a related marker for the vascular injury. ${ }^{29}$ Final results were expressed as $\mathrm{ng} \mathrm{mL}^{-1}$.

\section{Biochemical parameters including inflammatory cytokine}

After the animals were submitted to euthanasia, blood was collected as previously described to detect the tumour necrosis factor $\alpha$ (TNF- $\alpha$ ) and interleukin $1 \beta$ (IL-1 $1 \beta$ ) levels with ELISA kits for systemic inflammation. All indexes were operated using commercial colorimetric enzymatic diagnostic kits.

\section{Statistical analysis}

In our experiments, the data were expressed as mean $\pm \mathrm{SD}$ (standard deviation). All statistical analyses and graphs were performed using the Graph Pad Prism 5 software. Statistical evaluation of the results was performed using one-way analysis of variance by using the SPSS 19.0 system. Values of $p=0.05$ or less were considered to be significant.

\section{Results}

\section{Effects of apigenin on percentage of body fat and blood pressure}

Oral administration of apigenin reduced the percentage of body fat significantly versus that observed for the model group treated with fructose by the end of the experiment $(2.36 \pm 0.06 \% v s$. $3.59 \pm 0.31 \%, P<0.05$, Fig. 2A). Apigenin (50 mg kg $\mathrm{mg}^{-1}$ ) decreased SBP from $146 \mathrm{mmHg}$ to $123 \mathrm{mmHg}$ at the end of twelve weeks when compared with that in the model group $(P<$ 0.05 , Fig. 2B). These results showed that apigenin can decrease the elevated percentage of body fat and blood pressure induced by the excessive ingestion of fructose.

\section{Apigenin ameliorated insulin resistance in experimental rats}

At the end of the experiment, the serum was collected to measure the levels of glucose and insulin within 24 hours of sacrificing the rats. Homeostasis model assessment of insulin resistance (HOMA-IR) and Quantitative Insulin Sensitivity Check Index (QUICKI) were utilized to determine the sensitivity of insulin. Apigenin clearly improved the sensitivity of insulin in rats that had consumed high concentrations of fructose for twelve weeks (Fig. 2C and D).

\section{Effect of apigenin on lipid metabolism}

To evaluate the effect of apigenin on lipid metabolism, the levels of TG, TC and HDL-C were measured in serum within 48 hours after the experimental rats were sacrificed..$^{30}$ The TG level in the model group increased to $1.96 \pm 0.07 \mathrm{mmol} \mathrm{L}^{-1}$ as compared to that in the normal group $\left(0.79 \pm 0.13 \mathrm{mmol} \mathrm{L}^{-1}\right)$. Apigenin reduced the TG level significantly $(0.82 \pm 0.16 \mathrm{mmol}$ $\mathrm{L}^{-1}, P<0.01$, Fig. 3A). The levels of TC were increased by $10 \%$ fructose and were attenuated by apigenin $\left(2.13 \pm 0.06 \mathrm{mmol} \mathrm{L}^{-1}\right.$ vs. $1.60 \pm 0.11 \mathrm{mmol} \mathrm{L}^{-1}, P<0.01$, Fig. 3B). The concentration of the model group was lower than that of the normal group in terms of HDL-C $\left(0.73 \pm 0.12\right.$ vs. $\left.1.13 \pm 0.07 \mathrm{mmol} \mathrm{L}^{-1}\right)$, and apigenin could restore the decreased concentration (1.04 \pm $0.06 \mathrm{mmol} \mathrm{L}^{-1}, P<0.05$, Fig. 3C). These data indicated that apigenin significantly ameliorated disordered lipid metabolisms in rats fed with high amounts of fructose.

\section{Apigenin improved the pathological injury of thoracic aorta}

The thickness of the aortic wall was significantly higher as compared to that of the normal group, as indicated by hematoxylin-eosin staining. Apigenin decreased the thickness of the thoracic aorta near to the value of the control group $(P<$ 0.01, Fig. 4A and B). Meanwhile, EVG staining indicated that the structure of elastin was weaker and flattened in the model group versus that in the normal group. Apigenin could improve the wavy structure of elastin to some extent (Fig. 4C). In short, apigenin helped prevent vascular injury when rats were exposed to high fructose. 
A

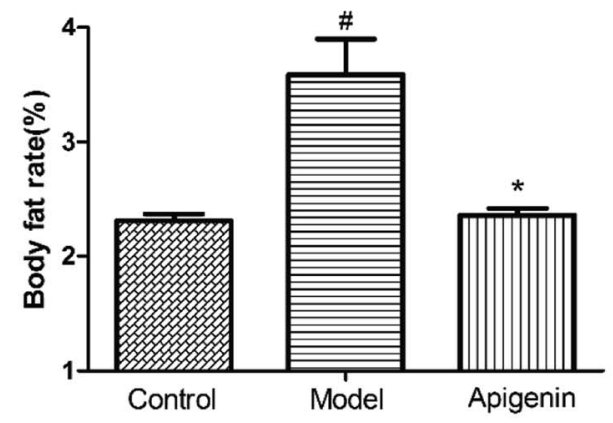

C

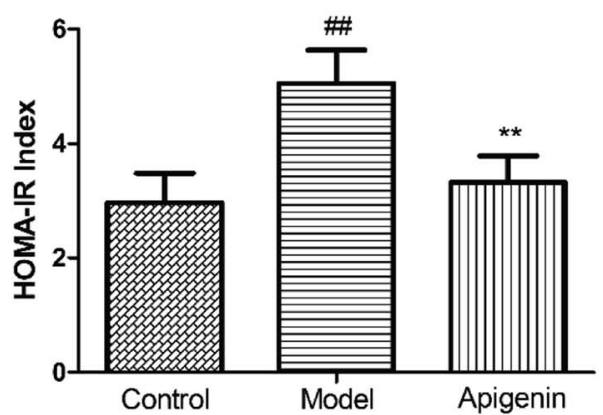

B

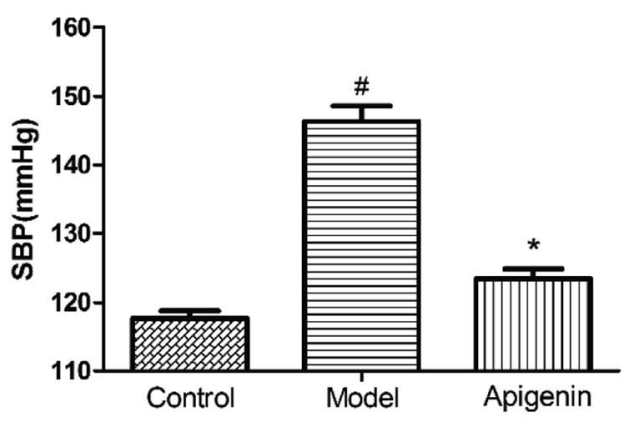

D

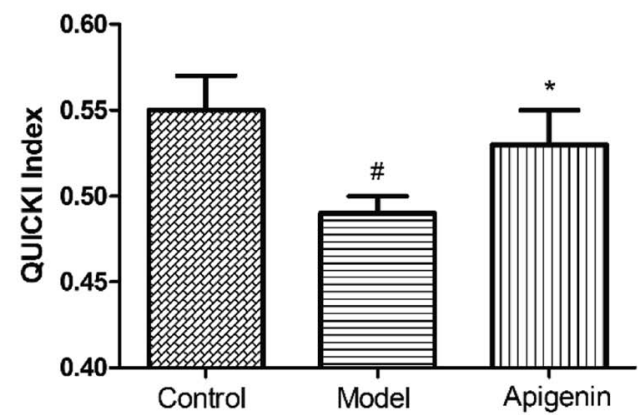

Fig. 2 Apigenin ameliorates the metabolic disorders induced by high fructose. (A) Percentages of body fat; (B) systolic pressure, SBP; HOMA-IR (C) and QUICKI index (D) were evaluated the level of insulin resistance of the rats. All the data are expressed as mean \pm SD $(n=8)$. ${ }^{\#} P<0.05,{ }^{\# \#} P<$ 0.01 compared to the normal group; $* P<0.05$ compared to the model group.

\section{Effect of apigenin on the expression of GLUT1 protein and} PI3K/AKT pathway

To explore whether the signalling pathway was inhibited by apigenin in experimental rats, we detected the total expressions of PI3K and AKT proteins of the thoracic aorta using western blot. As shown in Fig. 5A and B, fructose significantly increased the total PI3K expression as well as the level of AKT as compared to the observations for the normal group $(P<0.01$, Fig. $5 \mathrm{~B}$ and $D)$. The data showed that apigenin could reduce the upregulation of $\mathrm{PI} 3 \mathrm{~K}$ and $\mathrm{AKT}$ proteins as compared to that in the model group in the thoracic aorta. As shown in Fig. $5 \mathrm{E}$ and
F, the GLUT1 level in the model group was remarkably higher than that in the control group $(P<0.01)$. The data showed that apigenin down-regulated the expression of GLUT1 in thoracic aorta.

\section{Influence of apigenin on inflammation response system and ADMA}

We detected the levels of TNF- $\alpha$ and IL-1 $\beta$ to evaluate the immune system of rats using high levels of fructose and apigenin. ${ }^{31}$ As shown in Fig. $6 \mathrm{~A}$ and B, the levels of TNF- $\alpha$ and IL-1 $\beta$ both went up significantly in the model group as compared to
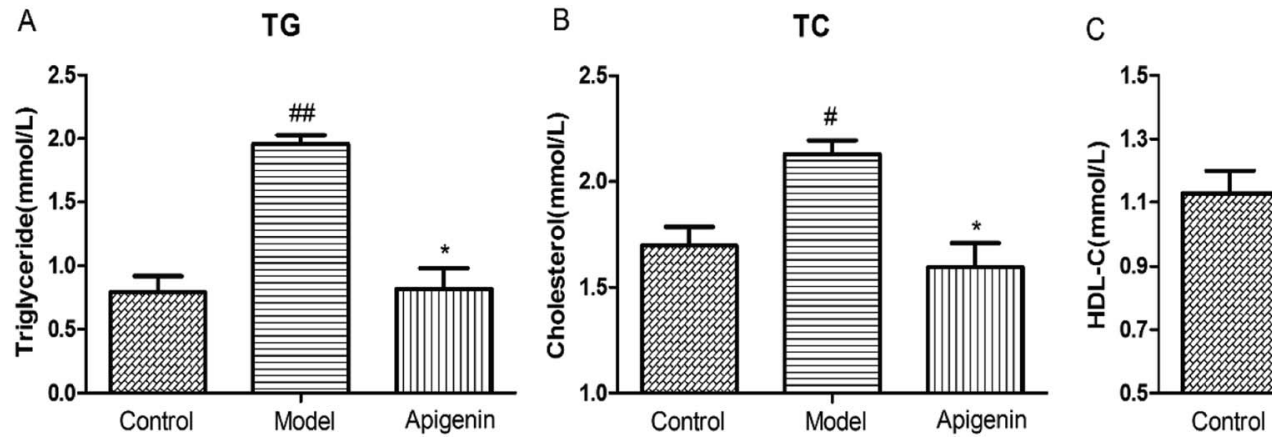

HDL-C

Fig. 3 Effects of apigenin on lipid metabolism from rats with metabolic disturbance: (A) the concentration of triglyceride (TG), (B) cholesterol (TC) level and (C) HDL-C level. All the data are expressed as mean $\pm \mathrm{SD}\left(n=8\right.$ rats). ${ }^{\#} P<0.05$, ${ }^{\# \#} P<0.01$ compared to the normal group; ${ }^{*} P<$ $0.05, * * P<0.01$ compared to the model group. 


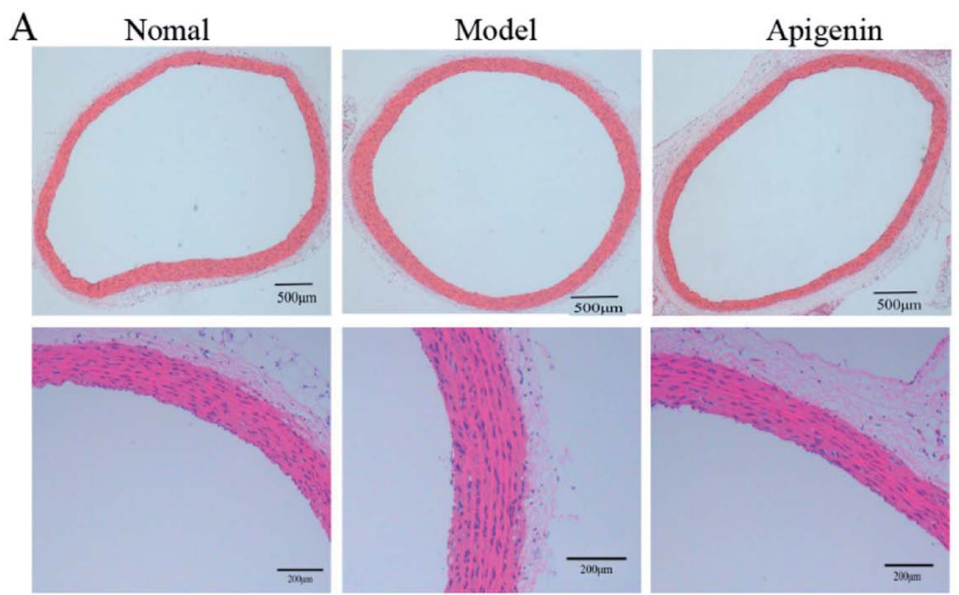

B

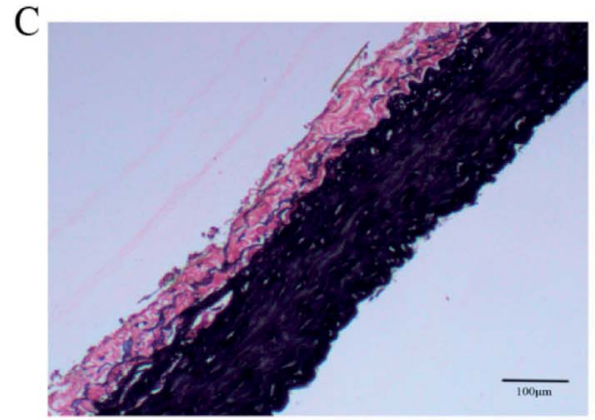

Nomal

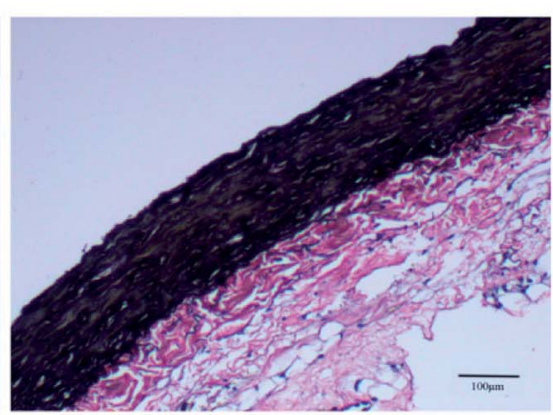

Model
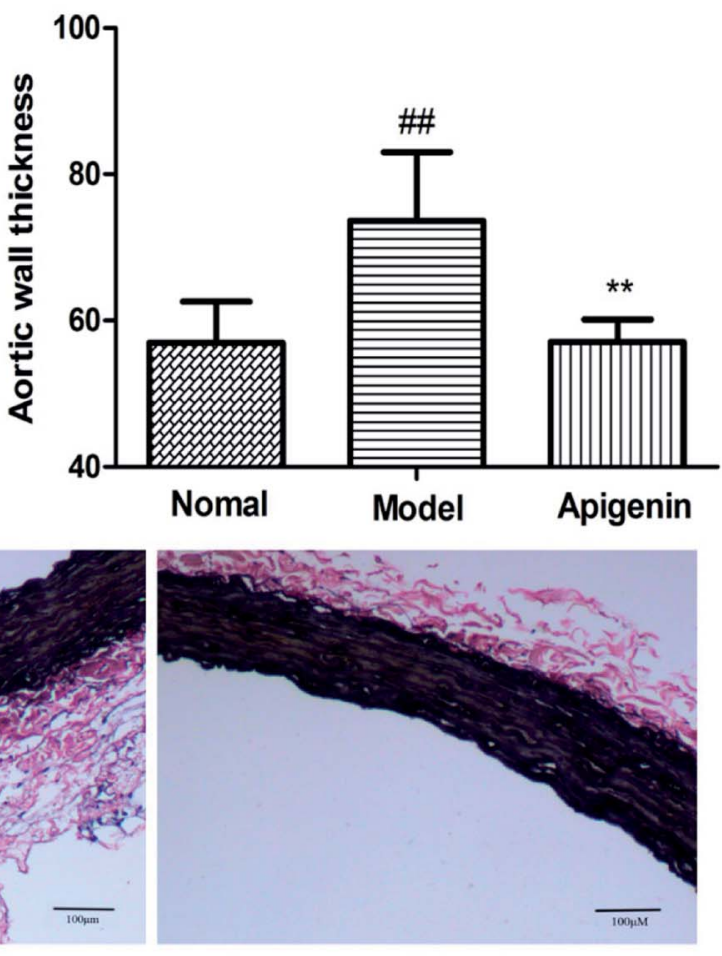

Apigenin

Fig. 4 Rats with metabolic disturbance showed the pathological injury of thoracic aorta by hematoxylin-eosin (HE) staining and EVG staining. (A) The hematoxylin-eosin (HE) staining of the vascular endothelium. (B) The calculation of average thickness of the media by the Image-Pro Plus software. (C) The changed elastic fibers are presented by EVG staining. All the data are expressed as mean \pm SD $\left(n=8\right.$ rats). ${ }^{\#} P<0.05,{ }^{\# \#} P<0.01$ compared to the normal group; $* P<0.05, * * P<0.01$ compared to the model group.

those in the control group. The apigenin treatment downregulated the increased levels $\left(232 \pm 16 v s .424 \pm 12 \mathrm{pg} \mathrm{mL}^{-1}\right.$, respectively, for TNF- $\alpha$; $174 \pm 13$ vs. $334 \pm 15 \mathrm{pg} \mathrm{mL}^{-1}$, respectively, for IL-1 $\beta)(P<0.01$, Fig. $6 \mathrm{~A}$ and $\mathrm{B})$. When the rats were sacrificed, the concentrations of ADMA in the serum were measured in time following the instructions. High ADMA levels are closely related to cardiovascular diseases, especially hypertension. ${ }^{32}$ The fructose group (model group) exhibited significantly increased level of ADMA $\left(187.5 \pm 6.5 \mathrm{ng} \mathrm{mL}^{-1}\right)$ versus the control group (156.9 $\left.\pm 2.6 \mathrm{ng} \mathrm{mL}^{-1}\right)$ (Fig. 6C).

\section{Discussion}

In this experiment, we evaluated the effects of apigenin on vascular injury and metabolic disorders in rats fed with high concentrations of fructose and revealed its underlying mechanisms. The data supported the following observations: (1) apigenin can prevent excessive-fructose-induced injury of blood vessels in rats; (2) apigenin effectively ameliorates vascular injury caused by high fructose, which might be related to PI3K/ AKT/GLUT1; (3) the pathway down-regulates the inflammation and ADMA to protect the thoracic aorta.

To the best of our knowledge, high concentration of fructose is currently one of the most commonly used models of metabolic disorders. ${ }^{33}$ In this experiment, we concluded that apigenin can prevent vascular damage to some extent in the high-fructose environment based on the observed results of decline in blood pressure, decreased levels of TG and TC, ameliorated pathological injury on thoracic aorta, and the increased level of HDL-C.

When fructose is largely absorbed into the blood, most of the fructose is metabolized by an enzymatic reaction. ${ }^{34}$ Therefore, the body protects itself through the promotion or inhibition of glucose transporter expressions under high fructose conditions. In modern life, due to the abuse of fructose as a sweetener, many people are deeply troubled by metabolic disturbances and complications. ${ }^{35,36}$ Studies have demonstrated that GLUTs are closely related to metabolic disorders. ${ }^{37-40}$

Apigenin is associated with anti-inflammatory effects, low blood pressure, and inhibition of tumour angiogenesis. ${ }^{41,42}$ In fact, the three hydroxyl groups at the 4,5 , and 7 positions determine these unique physiological effects of apigenin. ${ }^{43}$ PI3K/AKT proteins participate in the metabolic process. Apigenin can also improve the inflammation in hepatocytes and skeletal muscles. In our study, apigenin significantly reduced the vascular injury caused by high levels of fructose, as shown by HE and EVG staining; meanwhile, other metabolic markers such as blood pressure, percentage of body fat, insulin resistance and lipid metabolism were also ameliorated. Fundamentally, apigenin reduced the expressions of PI3K/AKT and 

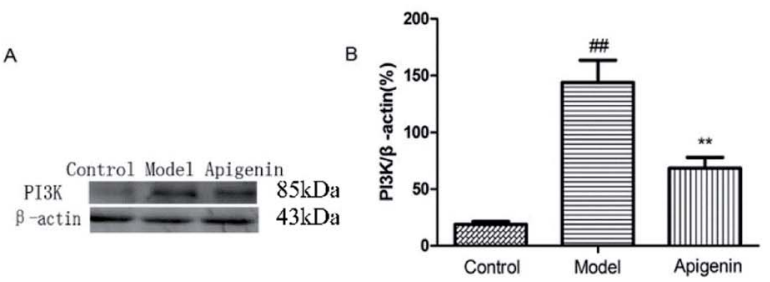

G
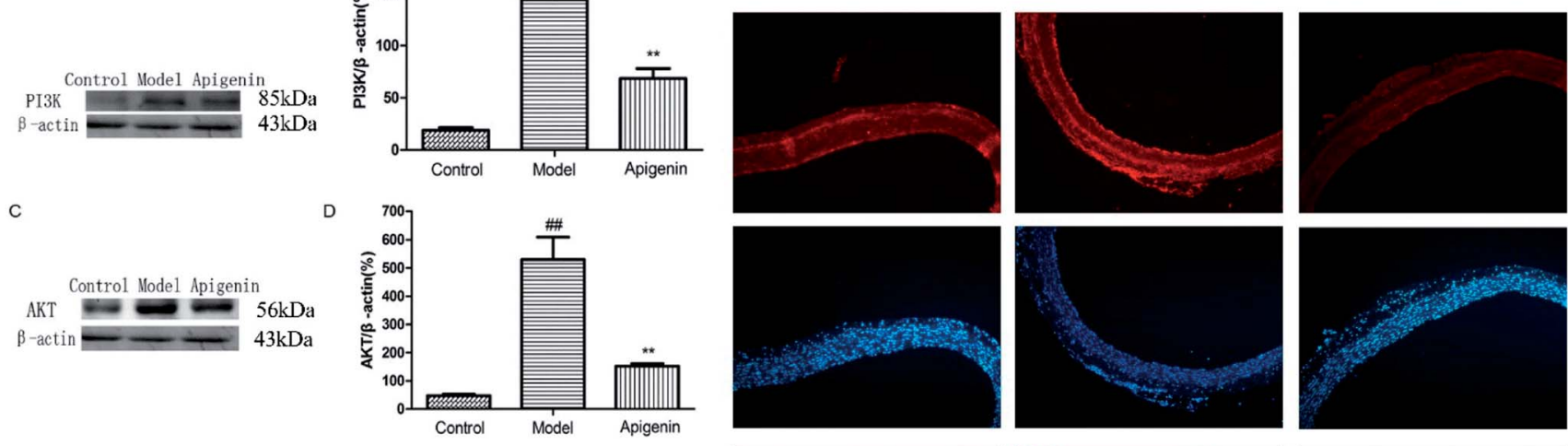

c

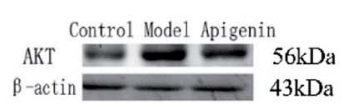

$56 \mathrm{kDa}$

E
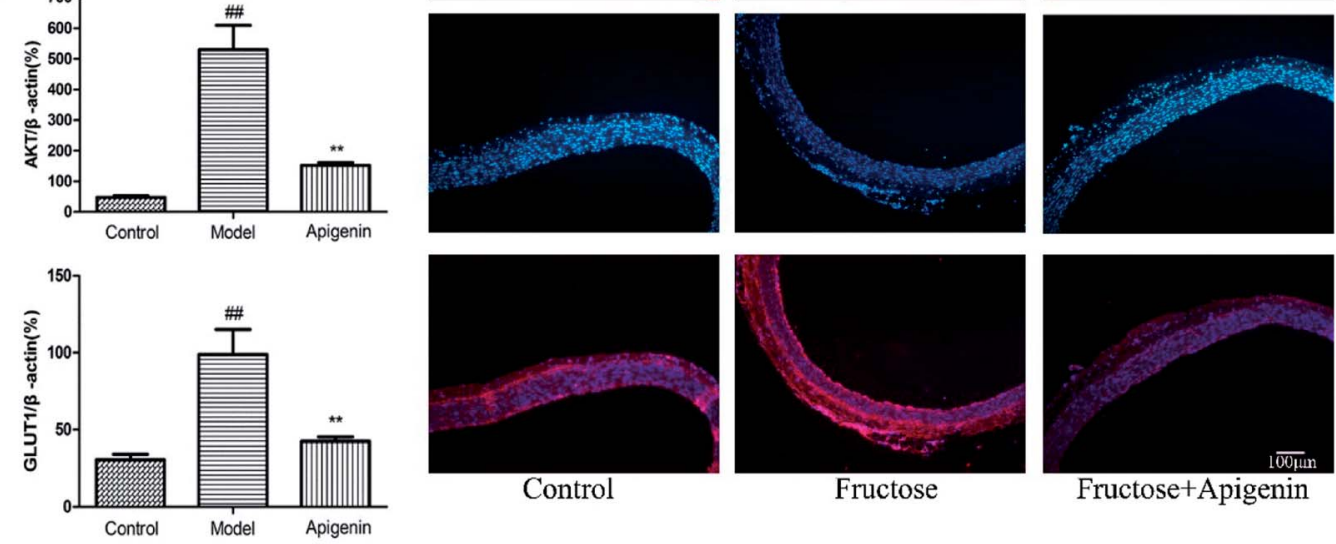

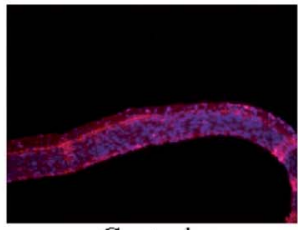

Control

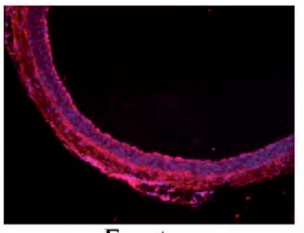

Fructose

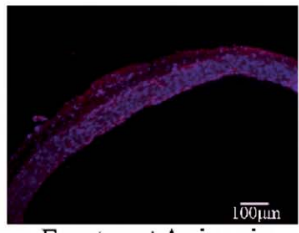

Fructose+Apigenin

Fig. 5 The expression levels of PI3K, AKT and GLUT1 were significantly higher in high-fructose-fed rats, and they decreased with apigenin consumption. The expressions of PI3K (A), AKT (C) and GLUT1 (E) proteins in the thoracic aorta. (B) The levels of PI3K were quantified by densitometry; (D) levels of AKT were quantified by densitometry; (F) levels of GLUT1 were quantified by densitometry. (G) GLUT1 was shown by Immunofluorescence staining. All the data are expressed as mean $\pm \mathrm{SD}$ ( $n=8$ rats). ${ }^{\# \#} P<0.01$ compared to the normal group; ${ }^{* * P}<0.01$ compared to the model group. Scale bar is $100 \mu \mathrm{m}$.

A

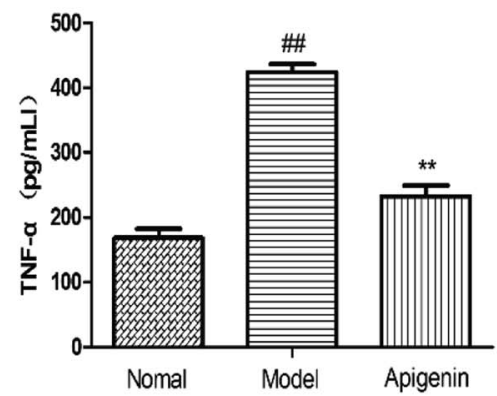

B

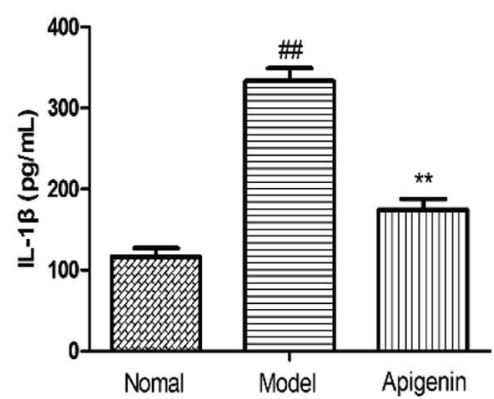

C

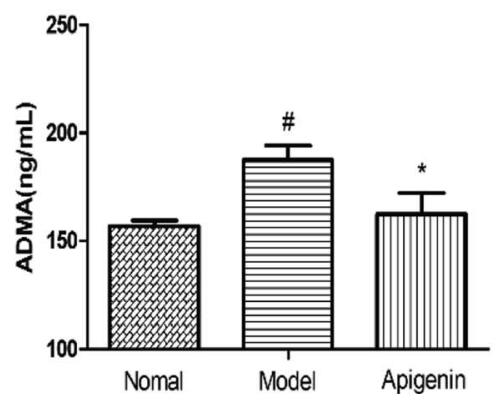

Fig. 6 The levels of immune factors and ADMA in high fructose-fed rats and groups with apigenin. (A) The levels of TNF- $\alpha$ and (B) IL-1 $\beta$ were detected by ELISA kits in serum ( $\mathrm{pg} \mathrm{mL}^{-1}$ ). (C) The ADMA levels $\left(\mathrm{ng} \mathrm{mL}^{-1}\right.$ ) are associated with the severity of cardiovascular diseases. All the data are expressed as mean \pm SD $\left(n=8\right.$ rats). ${ }^{\#} P<0.05,{ }^{\# \#} P<0.01$ compared to the normal group; $* P<0.05, * * P<0.01$ compared to the model group.

GLUT1 proteins of the thoracic aorta. Additionally, the levels of immune factors and ADMA increased in the damaged blood vessel and decreased in the apigenin group. Therefore, we considered that apigenin can protect against the damage to thoracic aorta caused by high levels of fructose.

In short, we demonstrated that apigenin improved the excessive-fructose-induced vascular damage, which may be related to the inhibition of the PI3K/AKT/GLUT1 signalling pathway. Moreover, the pathway further down-regulated the inflammation and ADMA level to protect the thoracic aorta. Above all, apigenin might be a potential candidate to develop new strategies to prevent vascular diseases.

\section{Conclusion}

These data indicated that excessive absorption of fructose may cause metabolic disorders and vascular injury in rats. Interestingly, apigenin can ameliorate the vascular injury in highfructose-fed rats. PI3K/AKT/GLUT1 pathway was downregulated; the levels of ADMA as well as the immune factors decreased. All these results may help push the very boundaries of our scientific knowledge concerning high-fructose-induced vascular injuries and finally open the path to discover both highly efficient and safe solutions to mitigate the vascular injury induced by high levels of fructose. 


\section{Author contributions}

Authors Chen, Tan and Ding mainly designed the study and interpreted the results. Tan performed the biochemical analysis of thoracic aorta and carried out the histopathological examination of thoracic aorta. Chen collected test data and drafted the final manuscript. All authors conducted the rats' experiments and approved the final manuscript.

\section{Conflicts of interest}

There are no conflicts to declare.

\section{References}

1 J. M. Rippe and T. J. Angelopoulos, Adv. Nutr., 2015, 6, 430439.

2 S. M. Alwahsh and R. Gebhardt, Arch. Toxicol., 2017, 91, 1545-1563.

3 M. J. Dekker, Q. Su, C. Baker, A. C. Rutledge and K. Adeli, Am. J. Physiol.: Endocrinol. Metab., 2010, 299, 685-694.

4 M. A. P. Mccullough, J. M. Johnson and J. A. Phillips, J. Food Sci., 1986, 51, 536-537.

5 L. M. Hanover and J. S. White, Am. J. Clin. Nutr., 1993, 58, 724.

6 C. M. Labak, P. Y. Wang, R. Arora, M. R. Guda, S. Asuthkar, A. J. Tsung and K. K. Velpula, Am. J. Cancer Res., 2016, 6, 1599.

7 V. Douard and R. P. Ferraris, Am. J. Physiol.: Endocrinol. Metab., 2008, 295, 227-237.

8 A. Scheepers, H. G. Joost and A. Schürmann, JPEN, J. Parenter. Enteral Nutr., 2004, 28, 364-371.

9 H. J. Martin, F. Kornmann and G. F. Fuhrmann, Chem.-Biol. Interact., 2003, 146, 225.

10 J. Zhang, Z. Deng, J. Liao, C. Song, C. Liang, H. Xue, L. Wang, K. Zhang and G. Yan, J. Cereb. Blood Flow Metab., 2013, 33, 567.

11 Y. Y. Bao, S. H. Zhou, Z. J. Lu, J. Fan and Y. P. Huang, Oncol. Rep., 2015, 34, 1805.

12 R. H. Böger and E. S. Ron, Altern. Med. Rev., 2005, 10, 14.

13 L. Orlandi, W. F. Fonseca, S. Enesmarques, V. A. Paffaro Jr, F. C. Vilela and A. Giustipaiva, J. Neuroimmunol., 2015, 289, 75-83.

14 X. Zhai, D. Ren, Y. Luo, Y. Hu and X. Yang, Food Funct., 2017, 8, 2536-2547.

15 T. P. Cushnie and A. J. Lamb, Int. J. Antimicrob. Agents, 2005, 26, 343-356.

16 Y. J. Moon, X. Wang and M. E. Morris, Toxicol. In Vitro, 2006, 20, 187-210.

17 R. M. van Dam, N. Naidoo and R. Landberg, Curr. Opin. Lipidol., 2013, 24, 25.

18 F. Ali, Rahul, F. Naz, S. Jyoti and Y. H. Siddique, Int. J. Food Prop., 2017, 20, 1197-1238.
19 S. Yano, D. Umeda, N. Maeda, Y. Fujimura, K. Yamada and H. Tachibana, J. Agric. Food Chem., 2006, 54, 5203-5207.

20 M. Leopoldini, I. P. Pitarch, A. Nino Russo and M. Toscano, J. Phys. Chem. A, 2004, 108, 92-96.

21 M. Karamese, H. S. Erol, M. Albayrak, G. F. Guvendi, E. Aydin and S. A. Karamese, Immunopharmacol. Immunotoxicol., 2016, 38, 228.

22 R. Bei, W. Qin, F. Wu, S. Wang, P. Cheng, L. Wang, B. Zeng, S. Ma and J. Liang, Eur. J. Pharmacol., 2016, 773, 13-23.

23 N. Zhang, X. H. Wang, S. L. Mao and F. Zhao, Molecules, 2011, 16, 3896-3907.

24 M. A. Vazquez-Prieto, C. Rodriguez Lanzi, C. Lembo, C. R. Galmarini and R. M. Miatello, J. Nutr. Metab., 2011, 2011, 475216.

25 Y. Sang, F. Zhang, H. Wang, J. Yao, R. Chen, Z. Zhou, K. Yang, Y. Xie, T. Wan and H. Ding, Food Funct., 2017, 8, 2331-2340.

26 M. Vogeser, D. König, I. Frey, H. G. Predel, K. G. Parhofer and A. Berg, Clin. Biochem., 2007, 40, 964-968.

27 P. A. Sarafidis, A. N. Lasaridis, P. M. Nilsson, M. I. Pikilidou, P. C. Stafilas, A. Kanaki, K. Kazakos, J. Yovos and G. L. Bakris, J. Hum. Hypertens., 2007, 21, 709-716.

28 C. Rukmini and T. C. Raghuram, J. Am. Coll. Nutr., 1991, 10, 593-601.

29 J. P. Cooke, Arterioscler., Thromb., Vasc. Biol., 2000, 20, 2032.

30 J. Li, X. Li, Z. Li, L. Zhang, Y. Liu, H. Ding and S. Yin, Food Funct., 2017, 8, 2886.

31 M. A. Jonker, J. L. Hermsen, Y. Sano, A. F. Heneghan, J. Lan and K. A. Kudsk, Surgery, 2012, 151, 278.

32 R. H. Böger, L. M. Sullivan, E. Schwedhelm, T. J. Wang, R. Maas, E. J. Benjamin, F. Schulze, V. Xanthakis, R. A. Benndorf and R. S. Vasan, Circulation, 2009, 119, 1592.

33 Y. J. Huang, V. S. Fang, C. C. Juan, Y. C. Chou, C. F. Kwok and L. T. Ho, Metabolism, 1997, 46, 1252-1258.

34 N. Adhikari, D. L. Basi, M. Carlson, A. Mariash, Z. Hong, U. Lehman, S. Mullegama, E. K. Weir and J. L. Hall, Arterioscler., Thromb., Vasc. Biol., 2011, 31, 86.

35 J. Klepper and T. Voit, Eur. J. Pediatr., 2002, 161, 295.

36 G. A. Bray, Diabetes Care, 2013, 36, 11-12.

37 J. P. Belman, E. N. Habtemichael and J. S. Bogan, Rev. Endocr. Metab. Disord., 2014, 15, 55-66.

38 E. Sato, A. Inoue, T. Kurokawa and S. Ishibashi, Brain Res., 1994, 637, 133.

39 Z. Y. Wen, Y. Wu, Y. Li, X. L. Chen, T. Wang, J. P. Ouyang and G. S. Li, Natl. Med. J. China, 2005, 85, 1460.

40 V. Douard and R. P. Ferraris, J. Physiol., 2013, 591, 401-414. 41 G. A. Naderi, S. Asgary, N. Sarraf-Zadegan and H. Shirvany, Mol. Cell. Biochem., 2003, 246, 193.

42 X. Yan, Q. Miao, P. Li, Y. Zhan and H. Shao, Cell Biosci., 2017, $7,50$.

43 Z. Ni, Mol. Simul., 2015, 41, 653-662. 